\title{
ПИКОСЕКУНДНАЯ ФЛУОРОМЕТРИЯ ПРОЦЕССА ДИФФУЗИИ ЭКСИТОНА В ПИГМЕНТНОМ АППАРАТЕ ХЛОРОПЛАСТОВ ВЫСШИХ РАСТЕНИИ
}

\section{Введение}

Одним из подходов к изучению диффузии экситона в некоторой матрице является измерение уменьшения длительности $\tau$ или квантового выхода $\varphi$ флуоресценции при мощном лазерном возбуждении. Действительно, при высоких энергиях в пределах радиуса межмолекулярного взаимодействия может возникнуть несколько синглетных (возможно, и триплетных) экситонов, аннигилирующих в результате бимолекулярных процессов $\left[{ }^{1-4}\right]$ :

$$
\begin{aligned}
& S_{1}+S_{1} \stackrel{v_{s s}}{\longrightarrow} S_{n}+S_{0} \rightarrow S_{1}+S_{0}+\text { тепло, } \\
& S_{1}+T_{1} \stackrel{v_{s T}}{\longrightarrow} S_{0}+T_{n} \rightarrow S_{0}+T_{1}+\text { тепло. }
\end{aligned}
$$

Здесь $S_{0}, S_{1}, S_{n}, T_{1}, T_{n}$ - основной, первый и $n$-й возбужденные синглетные и триплетные уровни молекулы, $\gamma_{S S}$ и $\gamma_{S T}$ - константы скорости синглет-синглетной $\left(S_{1}+S_{1}\right)$ и синглет-триплетной $\left(S_{1}+T_{1}\right)$ аннигиляций. Очевидно, процессы аннигиляции можно рассматривать как дополнительную дезактивацию возбужденного состояния, в результате чего происходит потеря кванта энергии и сокращаются $\tau$ и $\varphi$.

Имеются соотношения, связывающие константу аннигиляции $v$ с диффузионными параметрами - коэффициентом диффузии $D$, диффузионной длиной $L$ и вероятностью переноса возбуждения W. Так, для трехмерной изотропной диффузии при наличии лишь $S_{1}+S_{1}$-аннигиляции установлены следующие соотношения $\left[{ }^{5}\right]$ :

$$
\gamma_{\mathrm{SS}}=8 \pi D R, \quad L=\sqrt{D_{\tau}}, \quad W=D R^{-2} .
$$

Следовательно, если есть возможность экспериментально определить параметр $\gamma_{S S}$, то для некоторых приближений можно найти параметры $D, L$ и $\mathbb{W}$ и тем самым охарактеризовать диффузию возбуждения.

В случае $S_{1}+S_{1}$-аннигиляции общее выражение для изменения плотности синглетных экситонов имеет вид

$$
d n(t) / d t=c(t)-\beta n(t)-0,5 \gamma_{s s} n^{2}(t),
$$

где $c(t)$ - функция источника, $\beta=1 / \tau_{0}-$ константа скорости дезактивации в отсутствие $S_{1}+S_{1}$-аннигиляции. Решение уравнения (3) для трехмерной диффузии, когда плотность экситпнов в ходе аннигиляции убывает равномерно по объему, а сами аннигиляционные процессы начинаются после установления равномерного распределения возбуждения в объеме, т, е. $\gamma_{s s} n(t) \ll 1 / \tau_{\text {пр }}$, где $\tau_{\text {пр }}$ - среднее время прыжка, имеет вид 


$$
n(t)=\left[-\frac{\gamma_{S S}}{2 \beta}+\left(\frac{1}{n(0)}+\frac{\gamma_{S S}}{2 \beta}\right)^{-1}\right]
$$

Здесь $n(0)=\sigma N I-$ число поглощенных фотонов, $\sigma-$ сечение поглощения молекулы, $N$ - число молекул в объеме, $I$ - плотность энергии лазерного излучения. Из уравнения (4) легко получить выражения для относительного изменения квантового выхода $\varphi / \varphi_{0}$ и длительности $\tau$ флуоресценции от энергии возбуждающего импульса (под длительностью флуоресценции при неэкспоненциальном распаде будем подразумевать время, в течение которого населенность $S_{1}$-уровня падает в $e$ раз) $\left[{ }^{4}\right]$ :

$$
\begin{gathered}
\varphi / \varphi_{0}=\frac{1}{\beta} \ln \left\{\left[e+\frac{\gamma_{s s} n(0)}{2 \beta}\right] /\left[1+\frac{\gamma_{s S} n(0)}{2 \beta}\right]\right\}, \\
\tau=\frac{2 \beta}{\gamma_{s S} n(0)} \ln \left[1+\frac{\gamma_{s s} n(0)}{2 \beta}\right] .
\end{gathered}
$$

Именно уравнения (5) позволяют экспериментально определить $\gamma_{\text {SS. }}$ Для этого необходимо сравнить теоретические и экспериментальные зависимости $\tau$ и $\varphi / \varphi_{0}$ от энергии возбуждения для разных $\gamma_{S S}\left[{ }^{4,6}\right]$. Совершенно очевидно, что изложенный выше подход к анализу нелинейного тушения $\tau$ и $\varphi$ флуоресценции не исчерпывает все случаи переноса электронного возбуждения и должен быть существенно углублен и дополнен. Но тем не менее уже нынешнее состояние теории позволяет сделать определенные выводы о диффузии возбуждения в пигментном аппарате фотосинтезируюших организмов.

Другим эффективным подходом к изучению процессов диффузии возбуждения является изучение температурной зависимости $\tau$. Действительно, если перенос энергии связан с преололением энергетического барьера, то с понижением температуры колебательные уровни $S_{1}$-состояния будут «вымораживаться», вероятность переноса энергии падать, а $\tau$ возрастать. Показано $\left[{ }^{7}\right]$, что в таком случае температурная зависимость $\tau$ описывается уравнением

$$
\tau=\left(A+B \mathrm{e}^{-\Delta E / k T}\right) .
$$

Наконец, очень информативным является измерение $\tau$ флуоресценции различных пигмент-белковых комплексов (ПБК), образующих пигментный аппарат фотосинтезируюших организмов: комплекс светособирающей антенны (ССА). лишенной реакционных центров (РЦ) фотосистем I и II (ФС I и ФС II): субхлоропластные частицы, обогашенные РЦ I или РЦ II (частишы ФС I и частицы ФС II); комплексы собственно PЦ I и РЦ II с ближайшим пигментным окружением. Измерение $\tau$ флуоресценции таких препаратов позволяет определить константы межкомплексного переноса энергии.

Предметом данной работы является исследование диффузии возбуждения в пигментном аппарате хлоропластов методами импульсной флуорометрии с привлечением явления $S_{1}+S_{1}$-аннигиляции, температурных измерений и фрагментирования препаратов.

\section{Импульсный флуорометр}

Принципы импульсной флуорометрии и экспериментальная установка подробно описаны в $\left[{ }^{8}\right]$. Источником возбуждения является одиночный импульс света длительностью $\sim 2 n c$, генерируемый лазером на фосфатном стекле с неодимом. В случае необходимости одиночный импульс можно усилить в трехпроходном усилителе до энергии $\sim 50$ мДж. Бла- 
годаря последовательному преобраӟовагию диапазон длин волн возбуждения представлен линиями 530,353 и 265 нм.

В качестве регистратора в установке используется электронно-оптическая камера (ЭОК). Корректное проведение измерений требует контроля параметров импульса возбуждения в каждом опыте. С этой целью часть энергии одиночного возбуждающего импульса подается непосредственно на фотокатод ЭОК с некоторым фиксированным опережением относительно времени прихода флуоресценции. Этот относительно простой способ позволяет постоянно контролировать аппаратную функцию прибора $K(t)$ и обеспечивает возможность измерений относительных изменений квантового выхода флуоресценции образца.

Регистрация свечения экрана ЭОК осуществляется телевизионной камерой с видиконом типа кадмикон. В качестве промежуточного запоминающего элемента использован анализатор типа LP-4840, информация может быть воспроизведена монитором, выведена на двухкоординатный графопостроитель или введена в ЭВМ для обработки.

Такая фотоэлектрическая обработка свечения экрана ЭОК имеет ряд преимуществ перед фотографической. Наиболее важные из них линейность и беспороговый характер регистрации сигнала, сравнимого с шумами видикона, повышение чувствительности и отношения сигнал/ шум, существенное сокращение времени обработки. Немаловажно и то, что телевизионную технику регистрации можно использовать в установке, работающей в частотном режиме, что позволяет значительно повысить чувствительность и динамический диапазон регистрируемых сигналов.

\section{Экспериментальные результаты}

Логика экспериментов по исследованию первичных процессов фотосинтеза методом импульсной флуорометрии заключалась в следующем. Сначала исследовалась диффузия электронного возбуждения в ССА, лишенной РЦ ФС I и ФС II [ $\left.{ }^{9}\right]$. Затем изучалось перераспределение энергии, накопленной в ССА, между ФС I и ФС II. С этой целью были проведены измерения на частицах ФС II и на целых хлоропластах. На следующем этапе измеряли процессы миграции энергии к фокусирующей антенне (ФА) I и захвата возбуждения РЦ I. Методика приготовления ПБК для таких целей описана в [ $\left.{ }^{10}\right]$. Наконец, представляется интересным исследовать роль длинноволновых форм хлорофилла (Хл), составляющих ближайшее пигментное окружение РЦ I и РЦ II.

При выполнении экспериментов на препаратах ССА установлено (рис. 1), что при изменении плотности фотонов в возбуждающем импульсе от $3 \cdot 10^{12}$ до $10^{16}$ фотон/см ${ }^{2} \tau$ сокращается от 2,5 до 0,15 нс. Как и следует из выражения (5), при высоких уровнях энергии возбуждения кривая затухания флуоресценции носит неэкспоненциальный характер.

При анализе данных рис. 1 прежде всего обращает на себя внимание то, что $\tau$ флуоресценции ССА при отсутствии аннигиляционных процессов составляет 2.5 нс, т. е. мало отличается от $\tau$ для разбавленных растворов Хл $\left[{ }^{11,12}\right]$. Этот факт позволяет сделать вывод об отсутствии значительных эффектов концентрационного тушения флуоресценции Хл $a$ in vivo, хотя в растворах с ростом концентрации эффект тушения играет определяющую роль в сокращении $\tau\left[{ }^{13}\right]$. Очевидно, отсутствие концентрационного тушения флуоресценции Хл $a$ in vivo обусловлено спецификой расположения молекул и их взаимодействием с белками на мембране.

Сравнение аналитических и экспериментальных зависимостей $\tau$ и $\varphi / \varphi_{0}$ (рис. $\left.1, a, \sigma\right)$ дает значение константы скорости $S_{1}+S_{1}$-аннигиляции $\gamma_{S S} \simeq 8 \cdot 10^{-8} \mathrm{~cm}^{3} \cdot \mathrm{c}^{-1}$. Используя соотношения (2), можно определить значения $L, D$ и $W: L \simeq 900 \AA, D \simeq 2 \cdot 10^{-2} \mathrm{Cm}^{2} \cdot \mathrm{C}^{-1} ; W \simeq 10^{12} \mathrm{c}^{-1}$. В pac- 

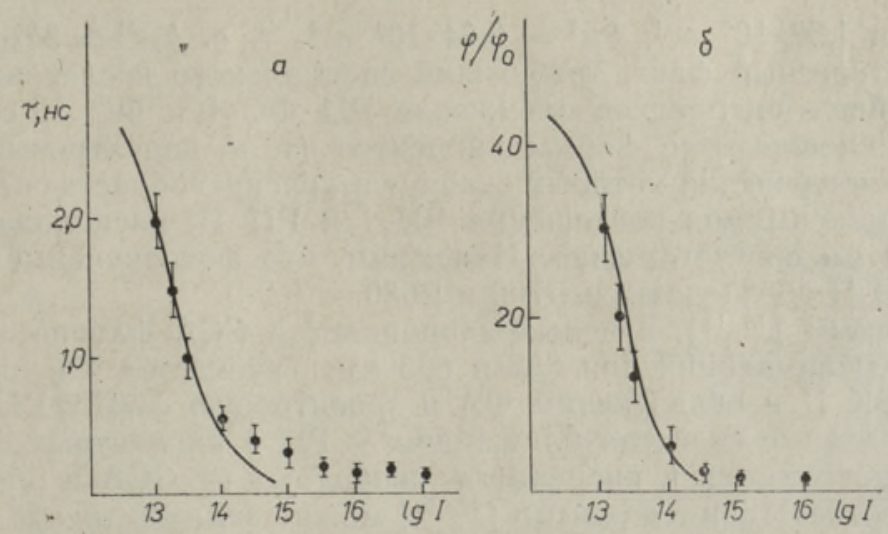

Рнс. 1. Зависимость длительности (a) и квантового выхода (б) флуоресценции светособирающей антенны от энергии одиночного лазерного импульса, $\lambda=535$ нм. Кривые построены по формулам (5)

четах эффективный радиус взаимодействия экситонов полагали равным 20^. Отметим высокие значения параметров $L, D$ и $W$. Величина, обратная вероятности переноса $\tau=1 / W=10^{-12} c$, означает время жизни возбуждения на отдельной молекуле. Из спектральных характеристик Хл а для двухуровневой системы можно оценить энергию взаимодействия, приводящую к сокращению $\tau$ от $5 \cdot 10^{-9}$ (in vitro) до $\sim 10^{-12}$ c. Расчеты показывают, что энергия межмолекулярного взаимодействия Хл $a$ in vivo $E_{1-2} \leqslant 10 \mathrm{~cm}^{-1}$. Исходя из значений энергии межмолекулярного взаимодействия $\left[{ }^{14}\right]$ и параметров диффузии возбуждения [ $\left.{ }^{5}\right]$ можно сделать вывод, что перенос энергии возбуждения между молекулами Хл $a$ CСА осуществляется локализованными экситонами.

Рассмотрим дальнейшие пути эволюции электронного возбуждения, возникшего в ССА. Поскольку ССА хлоропластов высших растений обеспечивает энергией ФС I и ФС II, представляется интересным определить константы скорости переноса энергии от ССА на ФА I и ФА II.

Были выполнены измерения $\tau$ на частицах ФС II и целых хлоропластах. С энергетической точки зрения частицы ФС II можно рассматривать как ССА, к которой присоединены ФА ІІ с РЦ II (ПБК II). Следовательно, изменение $\tau$ флуоресценции ССА в отсутствие аннигиляции будет отражать влияние ПБК II, играющего роль ловушки. Целые хлоропласты можно рассматривать как ССА, к которой присоединены ПБК II и ПБК I, и изменение $\tau$ отражает влияние уже двух типов ловушек.

Установлено, что в области значений интенсивности возбуждаюшего света $I \leqslant 5 \cdot 10^{14}$ фотон $/$ см $^{2}$ в пределах точности измерений $(\sim 10 \%)$ $\tau$ ССА частиц ФС II и целых хлоропластов постоянно и составляет 450 и 350 nс соответственно. Логично предположить, что сокращение $\tau$ CСА от $\sim 2,5 \mathrm{Hc}$ до $450 \mathrm{nc}$ и даже до $350 n c$ является следствием примесного тушения $\tau$ ССА ПБК II и ПБК I.

Константы миграции энергии от ССА на ПБК II $k_{\mathrm{M}}$ II и ПБК I $k_{\mathrm{M}}{ }^{\mathrm{I}}$ нетрудно определить из тривиальных соотношений

$$
k_{\mathrm{M}}^{\mathrm{II}}=1 / \tau(\text { Ф II })-1 / \tau(\mathrm{CCA}) ; \quad k_{\mathrm{M}}^{\mathrm{I}}=1 / \tau(\mathrm{X} л \Pi)-1 / \tau(\text { ФС II }) .
$$

Здесь $\tau($ ССА) - длительность флуоресценции пигментов ССА, свобод-

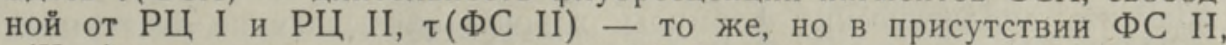
$\tau($ Хлп) - то же, но в присутствии ФС I и ФС II. Оценки показывают, 
что $k_{\mathrm{M}} \mathrm{II}=1,82 \cdot 10^{9} c^{-1}, k_{\mathrm{M}}^{\mathrm{I}}=0,64 \cdot 10^{9} c^{-1}$, т. е. $k_{\mathrm{M}}^{\mathrm{II}} \simeq 3 k_{\mathrm{M}^{\mathrm{I}}}$. Это довольно интересный факт, требующий специального исследования.

Ближайшее пигментное окружение РЦ ФС I и ФС II составляют ФА и длинноволновые формы пигментов (т. н. зацентровые формы), максимум поглощения которых сдвинут в красную область спектра относительно максимумов поглощения РЦ I и РЦ II, расположенных при 700 и 680 нм соответственно. Напомним, что фотоактивные пигменты РЦ I и РЦ II обозначаются Р700 и Р680.

По данным $\left[{ }^{15,16}\right]$, в целых хлоропластах ССА флуоресцирует при 680 нм, флуоресценция при 685 и 695 нм испускается Хл, входящим в состав ПБК II и образующим ФА и зацентровую форму РЦ II.

Спектральные свойства ближайших к РЦ I пигментных форм Хл $a$ хорошо проявляются в препаратах, свободных от ССА и обогащенных P700. В соответствии с данными $\left[{ }^{15-18}\right]$ можно заключить, что ФА І представлена двумя формами Хл, поглощающими при 682 и. 690 нм (Хлб82 и Хл 690$)$. Флуоресценция ФА I сосредоточена в основном в области 694 нм. Спектр зацентрованных форм РЦ I представлен формами Хл, флуоресцирующими при 720,732 и, возможно, при 750 нм (Хл ${ }^{720}, \mathrm{Xл}^{732}$ и $\left.\mathrm{Х}^{750}\right)$.

Таким образом, исследование флуоресценции ФА II и зацентровой формы РЦ II выполнялось на целых хлоропластах, свечение регистрировалось при 685 и 695 нм. Такое же изучение ближайших пигментных форм РЦ I проводили на ПБК I с отношением Р700: Хл 2120 . Флуоресценцию регистрировали при 695 нм (ФА I) и 720,735 и 750 нм (зацентровые формы). Полосы флуоресценции выделяли интерференционными светофильтрами, полуширина которых составляла $\sim 10$ нм.

Установлено, что $\tau$ ФА І с активными РЦ равняется $\sim 30$ nc и не зависит от температуры. При окислении РЦ раствором $\mathrm{K}_{2} \mathrm{Fe}(\mathrm{CN})_{6} \tau$ ФA I возрастает до 60 nc. Сокращение $\tau$ Хл $a$, входящего в ФА I, более чем в 100 раз по сравнению с $\tau$ ССА можно объяснить миграцней энергии на Р700 с константой скорости $k_{\mathrm{M}}^{\Phi \mathrm{AI}} \simeq 1 / \tau \simeq 3,3 \cdot 10^{10} \mathrm{c}^{-1}$

Измерения температурной зависимости - $\tau$ Хл $a$, локализованного в ФА II $\left(\lambda_{\text {per }}=685\right.$ нм), показали, что $\tau$ полосы 685 нм $\left(\tau_{685}\right)$ практически не меняется при понижении температуры от 293 до 77 К и остается равным 350 nc.

Значение $\tau$ ФА II позволяет определить константу скорости $k_{\mathrm{M}}^{\Phi \mathrm{AII}} \simeq 1 / \tau_{685} \simeq 3 \cdot 10^{9} \tau^{-1}$, а значения $\tau$ ФА І и ФА II, кроме того, - и константы скорости деления зарядов в РЦ I и РЦ II $k_{s}$ I и $k_{e}$ II $\left[{ }^{19}\right]$, если считать квантовый выход деления зарядов $\varphi_{e} \simeq 0,9\left[{ }^{20}\right]$. Оказывается, что $k_{e} \mathrm{I} \simeq 1,5 \cdot 10^{11} c^{-1}, k_{e} \mathrm{II} \simeq 1,7 \cdot 10^{10} c^{-1}, \varphi$ флуоресценции Р700 и P680 составляют $3 \cdot 10^{-3}$ и $3 \cdot 10^{-4}$ соответственно. Иными словами, время жизни возбужденного состояния $\mathrm{P}^{*} 700 \tau_{700} \simeq 5-7 n \mathrm{c}, \tau_{680} \simeq 60 \mathrm{nc}$.

Температурные зависимости $\tau$ флуоресценции зацентровых форм

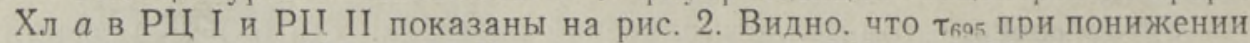
температуры от 293 до $77 \mathrm{~K}$ увеличивается от 350 до 1200 ne, $\tau_{720}$ - от 30 до 1700 nc, $\tau_{73}$ — от 30 до 2200 nc. При температvрах ниже $180 \mathrm{~K}$ обнаруживается разгорание флуоресценции полос 720,732 и 750 нм, причем для всех полос $\tau_{\text {разг }} \simeq 30$ nc, что отражает перенос энергии возбуждения от ФА I на Хл ${ }^{720}$, Хл$^{732}$ и Х Хл ${ }^{750}$ с константой $k_{\mathrm{M}}^{c_{\mathrm{I}}} \simeq 3,3 \cdot 10^{10} c^{-1}$. Было установлено, что в пределах ошибок эксперимента температурные зависимости $\tau_{732}$ и $\tau_{750}$ совпадают, поэтому анализ мы будем проводить для полос флуоресценции 695,720 и 732 нм.

Анализ данных по изменению $\tau_{695}, \tau_{720}$ и $\tau_{732}$ от температуры (рис. 2 ) показывает, что подбором параметров $A, B$ и $\Delta E$ в формуле (6) можно получить аналитические формулы, хорошо описывающие экспериментальные результаты: 
$k_{\mathrm{M}^{\mathrm{I}}} \simeq 0,6 \cdot 10^{9} \mathrm{c}^{-1}$ и $k_{\mathrm{M}}^{\mathrm{Ii}} \simeq 2 \cdot 10^{9} \mathrm{c}^{-1}$. Затем энергия от $\Phi \mathrm{A}$ І и $\Phi \mathrm{A}$ II переносится на РЦ I и РЦ II с константами $k_{\mathrm{M}}^{\Phi \mathrm{I}} \simeq 3,3 \cdot 10^{10} c^{-1}$ и $k_{\mathrm{M}}^{\Phi \mathrm{II}} \simeq 3 \cdot 10^{9} \mathrm{C}^{-1}$. Разделение зарядов в РЦ I и РЦ ІІ осуществляется за $3-5$ и $60 n c$ соответственно. Альтернативой переносу энергии на РЦ I и РЦ II является перенос на зацентровые формы хлорофилла C-710, С-720 и C-685 соответственно. Эти формы также могут участвовать в обеспечении энергией РЦ, однако другим их назначением, и, по нашему мнению, более отвечающим действительной роли этих форм, является некая защитная функция, предотвращающая пигменты фотосинтетического аппарата от деструктивного окисления. Разумно предположить, что в условиях активного фотосинтеза дипольные моменты Хл $a$ ФА и РЦ имеют одинаковые направления, а момент Хл $a$ зацентровых форм ориентирован перпендикулярно. Тогда вся энергия возбуждения будет попадать на РЦ, а $\tau$ молекул-переносчиков будет малым и вероятность реакции с синглетным кислородом также мала. При окислении РЦ на мембране возникает электрическое поле $\sim 10^{5} \mathrm{~B} / \mathrm{cm}$. Возможно, в результате взаимодействия этого поля с дипольными моментами Хл $a$ ФА и зацентровых форм происходит поворот моментов и возникает межмолекулярное диполь-дипольное взаимодействие, в результате которого энергия от ФА эффективно передается Хл $a$ зацентровых форм.

Зацентровые формы Хл $a\left(\right.$ Хл$\left._{685}^{695}, \mathrm{Xл}_{710}^{720}, \mathrm{Xл}_{720}^{735}\right)$ наиболее длинноволновые и, по данным спекंтроскопии комбинационного рассеяния $\left[{ }^{21}\right]$, являются агрегатами нескольких молекул. В таких агрегатах велика роль концентрационного тушения $\left[{ }^{13}\right]$, приводящего к сокращению $\tau$ от наносекунд (мономеры) до десятков пикосекунд. Таким образом, когда РЦ окислены, энергия возбуждения эффективно передается зацентровым формам Хл $a$, где она быстро разменивается в тепло. Такой механизм функционирования пигментного аппарата может предотвращать фотодеструкцию молекул.

\section{ЛИТЕ РА Т У РА}

1. M a u zer a 11, D., Biophys. J., 16, № 1, 87-91 (1976).

2. Swenberg, C. E., Ge a cintov, N. E., Pope, M., Biophys. J., 16, № 12, $1447-1452$ (1976).

3. Geacintov, N. E., Bret on, J., S we n berg, C. E., Pallot in, G., Photochem, Photobiol,, 26, № 6, 629-638 (1977).

4. Рубин Л. Б., Корватовский Б. Н., Брагинская О. В., Па щенко В. 3., Першке Х., Тусов В. Б., Молекуляр. биол., 14, вып. 3 , $675-684(1980)$.

5. Агр а н о в и ч В. М., Теорня экситонов, М., «Наука», 1968.

6. Campillo, A. J., Shapiro, S. L., Kollman, V. H., Winn, K. R., Hy e r, R. C., Biophys. J., 16, № 1, 93-97 (1976).

7. Тусов В. Б., Корватовский Б. Н., Пащенко В. 3., Рубин Л. Б., Докл. АН СССР, 252, № 6, 1500-1504 (1980).

8. П а ще н ко В. З., Р у би н Л. Б., Квант. электроннка, (в печати).

9. Гуляев Б. А., Т етенькнн В. Л., Померанцев а О. М., Докл. АН СССР, 248, № 3, 752-755 (1979).

10. Thor n ber, J., Ann. Rev. Plant. Physiol., 26, 127 (1975).

11. B rody, S. S., Rabi now itch, E., Science, 125, № 3247,555 (1957).

12. Пащенко В. З., Р убин Л. Б., Рубин А. Б., Т усов В. Б., Фролов В. А., Ж. техн. физ., 45, № 5, 1122-1127 (1975).

13. Shapiro, S. L., Kollman, V. H., Campil1o, A. J., FEBS Lett., 54, № 3, $358-362(1975)$.

14. Зенькевич Э. И., Л осев А. П., Гурин о вич Г. П., Миграция энергии между пигментами фотосинтетического аппарата растений, Минск, АН БССР, 1971 .

15. Rijgersberg, C. P., Amesz, J., Thielen, A. P. G. M., Swager, J. A., Biochim. Biophys. Ácta, 545, № 3, 473-482 (1979).

16. Govind jee, Ya n g, L., J. Gen. Physiol., 49, 763-780 (1966).

17. Cho, F., Govind je e, Biochim. Biophys. Acta, 216, № 1, 139-150 (1970). 
18. Kochubei, S. M., Guliev, F. A., Photosynthetica, 14, № 2, 182-188 (1980). 19. Корватовский Б. Н., Кукарских Г. П., Т усов В. Б., Пащенко В. З., Р у б и н Л. Б., Докл. АН СССР, 253, № 5, 1251-1255 (1980).

20. Shuvalov, V. A., Klevanik, A. V., Sharkov, A. V., Kryukov, P. G., Ke, B., FEBS Lett., 107, № 2, 313-316 (1979).

21. Гаджиев 3. И., Годжаев Н. М., Пащенко В. 3., Рубин Л. Б., Чу р и н А. А., Докл. АН СССР, (в печати).

Московский государственный университет

им. М. В. Ломоносова

\section{B. RUBIN, V. Z. PASTSENKO}

\section{KORGEMATE TAIMEDE KLOROPLASTIDE PIGMENDIAPARAADIS TOIMUVA EKSITONI DIFUSIOONIPROTSESSI PIKOSEKUNDFLUOROMEETRIA}

Uurimus käsitleb ergastuse difusiooniprotsessi kloroplastide pigmendi-va'gukompleksides. Singlett-singlett-đ̊nnihilatsiooni kasutades on määratud ergastuse difusiooni karakteristikud valgust koguvas antennis.

On kindlaks tehtud fotosüsteemide I ja II valgust koguvalt antennilt fookustavatele antennidele suunatud energiaülekande kiiruskonstandid, mõõdetud fookustavate antennide fluorestsentsi kestust ning hinnatud laengute eraldamise kiirust reaktsioonitsentrites I ja II. Mōōtmistulemuste alusel on koostatud kõrgemate taimede fotosünteesi funktsionaalne skeem.

\section{B. RUBIN, V. Z. PASCHENKO}

\section{PICOSECOND FLUOROMETRY OF THE EXCITON DIFFUSION PROCESS IN CHLOROPLAST PIGMENT APPARATUS OF HIGHER PLANTS}

Results of the investigation of the excitation diffusion process in pigment-protein complexes of chloroplasts are reported. By the singlet-singlet annihilation fenomenon parameters characterizing excitation diffusion in light-harvesting antenna have been obtained: diffusion length $L \simeq 900 \AA$, diffusion coefficient $D \simeq 2 \cdot 10^{-2} \mathrm{~cm}^{2} \mathrm{~s}^{-1}$, electron transfer probability $W \simeq 10^{12} \mathrm{~s}^{-1}$. The rates of energy transfer between the light-harvesting and focussing antennae of photosystems I and II have been determined to be $\approx 0.6 \cdot 10^{9} \mathrm{~s}^{-1}$ and $2 \cdot 10^{9} \mathrm{~s}^{-1}$, respectively. The fluorescence duration of the focussing antennae of photosystems I and II has been found to be $\sim 30$ and $350 \mathrm{ps}$, respectively. Charge separation rates in reaction centres I and II are $\sim 1.5 \cdot 10^{11} \mathrm{~s}^{-1}$ and $1.7 \cdot 10^{10} \mathrm{~s}^{-1}$. The temperature dependence of fluorescence duration for the bands at $695,720,732$, and $750 \mathrm{~nm}$ was measured. On the basis of measurements a functional scheme of higher plants photosynthesis has been drafted. 